\title{
Implementation of Affective Knowledge for any Geo Location Based on Emotional Intelligence using GPS
}

\author{
B. Asraf Yasmin, R. Latha, R. Manikandan
}

\begin{abstract}
Today, Digital world plays a vital role in new era of science and technology. The main objective of the proposed research work is to provide information about the particular location based on similes and not as a document. So that the customer or the user can predict information about the particular place from the existing customer. This Information of the particular place is categories based on the necessity of the user. This proposed work provides web service, where the user has to enable the geo location of the mobile device, based on the geolocation questionnaire will be asked to the customer randomly. These Emotional Intelligence service will provide information to the new user; based on the ranking provided by the existing user, based on affective and factual knowledge.
\end{abstract}

Keywords: Emotional Intelligence, Affective Knowledge, GPS, Factual knowledge.

\section{INTRODUCTION}

Emotional Intelligence here connotes to, the psychological expression of human beings that could act as an input for a computer program. Such an input in turn would trigger or activate the next process. This Emotional Intelligence could even be combed to control the application based on the user's emotions, such as joy, sorrow and cry etc. In other words, the capacity of an individual based on the emotions with different feeling is labeled appropriately and thus the guided or managed emotion is directed to achieve the appropriate goal. Emotional Intelligence had been designed for the scientific community created by Goleman in the year 1995. There are several models of Emotional Intelligence; Mixed Model is the combination of smiley faces and traits of Emotional Intelligence. Next model reflects skills and characteristics for leadership performance. According to Daniel Goleman, an American psychologist, Emotional Intelligence is a throng of various values that enhances self directive principles such as selfawareness, self -regulation, motivation, empathy and social skills.

\subsection{Affective Knowledge}

Revised Manuscript Received on September 10, 2019.

B. Asraf Yasmin, 1Research Scholar, Department of Computer Application St.Peter's Institute of Higher Education and Research, Chennai, Tamil Nadu, India.

(Email: asrafyasmin05@gmail.com)

Dr. R. Latha, Head, Department of Computer Science \& Application, St.Peter's Institute of Higher Education and Research, Chennai, Tamil Nadu, India.

(Email: latharamavel@gmail.com)

R. Manikanda 3Head, Department of Computer Science, The Quaide Milleth College for Men, Chennai, Tamil Nadu, India.

(Email: manisankar27@gmail.com)

\subsection{Emotional Intelligence}

Affective Knowledge includes emotions of an individual and its resulting expression in the forms of smiley faces which are captured and evaluated by multinomial distribution algorithm. This algorithm is based on machine learning, Lexical Methods or combination of both. Though many types of unique affective knowledge may be incurred, by progressing still into affective state recognition, this could be classified under two main categories as seen below. First comes, the hash tag indicators of emotions which can be used to learn from the tweets or smiley faces. This hash tag indicator is mainly used in the research with generalized pattern of hash tag and phrases which represent emotion of the writers in tweets. Bootstrapped algorithm is used for learning its further categorization which would typecast the terms of Anger, Fear, Joy, Sadness, disappointment etc. Second is the understanding of those smiley faces by making a comparative study. This method segregates them into positive and negative expressions based on the affective knowledge.

\subsection{Factual Knowledge}

Factual Knowledge is the one that had been generated from linked data source which hold information about different products, which includes its characteristics, and few other details that fringes or elaborates it. Many people by default express their varying emotions as per their affective states in the form of smiley faces.

\subsection{Multinomial Distribution}

Multinomial Distribution is the general form of distribution based on different category with $\boldsymbol{n}$ independent trial which leads to success for the exactly $\boldsymbol{k}$ categories with each category having a given fixed probability of success. In other words, multinomial distribution gives the success rate for various categories that falls under a particular combination.

\subsection{Map Matching Algorithms}

Map Matching Algorithms are classified into four groups: a) Geometric b) Topological c) probabilistic and d) Other Advanced Techniques. The geometric based algorithm is mainly focused on a basic concept such as point to point matching, point to curve matching, curve to curve matching and also improved geometric map matching. Point to point and point to curve matching makes an ambiguous use of historical information. Curve to curve matching constructs piecewise curves from linear paths which are generated 
from the candidate nodes. Point to point produces some unpredicted and unexpected results.

\section{LITERATURE REVIEW}

In this survey, Emotional Intelligence is technically used to produce the output of the customer or user in the form of smiley rather than the text. The Emotional Intelligence can be predicted via Multinomial Distribution Algorithms. These aspects can be implemented in the real world using smart mobile application during navigation. Global Position System (GPS), technology is used to find the current location of the smart phone or particular digital device with help map matching algorithm. This survey aims to put forth a principle in which, emotional intelligence plays a vital role in opinion ranking and predict the necessary information which acts as services for the customer in the real digital world.

The literature survey gives the following outcome:

Emotional Intelligence can be classified into two : a) Affective Knowledge, b) Factual Knowledge

- Affective Knowledge mainly focuses on the sentimental analysis of the user or the customer that reflects in terms of document, based on the opinion mining.

- Factual Knowledge focus on the exiting data source and produce result in terms of rating based on the review given by the user.

- Map matching algorithms are of two categories :

a. Real time system to detect the physical location and marking its corresponding digital location online while connected to the network.

b. Stored data are processed and used accordingly while offline.

- Map Matching algorithms are classified as Macroscale, Microscale and Mesoscale.

- Map Matching Algorithms are categorized into Geometric, Topological, Probabilistic and other advanced techniques.

- Though Map Matching Algorithm supports various intelligent transport system application such as traffic, congestion etc. Big data challenges, characterization and security issues are noticed.

- Global Position System is used to track and monitor the position of any particular vehicle.

- GPS monitors by four satellites simultaneously while moving from one position to another position.

- Multinomial Distribution is mainly focused on probability of emotion with $\boldsymbol{m}$ number of categories from a particular location for $\boldsymbol{n}$ number of times which is used to estimate the opinion ranking for specific locations.

- Number of success predicted for the various categories leads to probability of particular combination which are captured up as results for multinomial distribution.
- A Multinomial Distribution will produce various outputs which are categorized by the expressions seen on different smiley faces, whereas binomial distribution will produce an output that has only two options as success or failure.

\section{OBJECTIVES OF RESEARCH}

The objective of this research work is to provide the information about the particular location based on smiley faces and not the documents. Data thus collected and stored could be of a great hint for the new users. The information of any particular place is categorized based on the necessity of the user.

The objectives of this research work are:

- To design an unique smart application for mobile phones, where the user would have to get authenticated by one-time registration by providing appropriate details as confidentiality is to be maintained.

- User has to enable the geo location of the mobile device, based on which questionnaire would be put forth to the customer randomly.

- Highly flexible as GPS would track the user's location though on move. Probability questionnaire would also be presented.

- This research work takes up the help of multinomial distribution algorithm to predict the ranking of any specific location based on categories got via smiley faces.

- Maintains security and flexibility whereby the user's personal information is stored under high secrecy and the location though quickly detected not let known to any other person.

- Ensures to overcome the disadvantages of RFID and Wi-Fi while being implemented.

- Provides accuracy and effective implementation of an application

- This Emotional Intelligence service will provide the information to the new user as per the ranking provided by the existing user based on affective and factual knowledge.

- Application would initially try to understand the user's interest to answer few questions as a feedback on any particular location which would then be manipulated using trait anxiety based on the affective knowledge

- In every 30 days information of the appropriate ranking based on the existing user's response.

\section{DESCRIPTION OF RESEARCH WORK}

The main objective of this research is to provide a service to the new user who enters, any particular location by grasping the feedback provided by the then existing users in the form of emoticons, that are analyzed on the basis of affective and factual knowledge. The design of the framework is as shown in figure 1. 


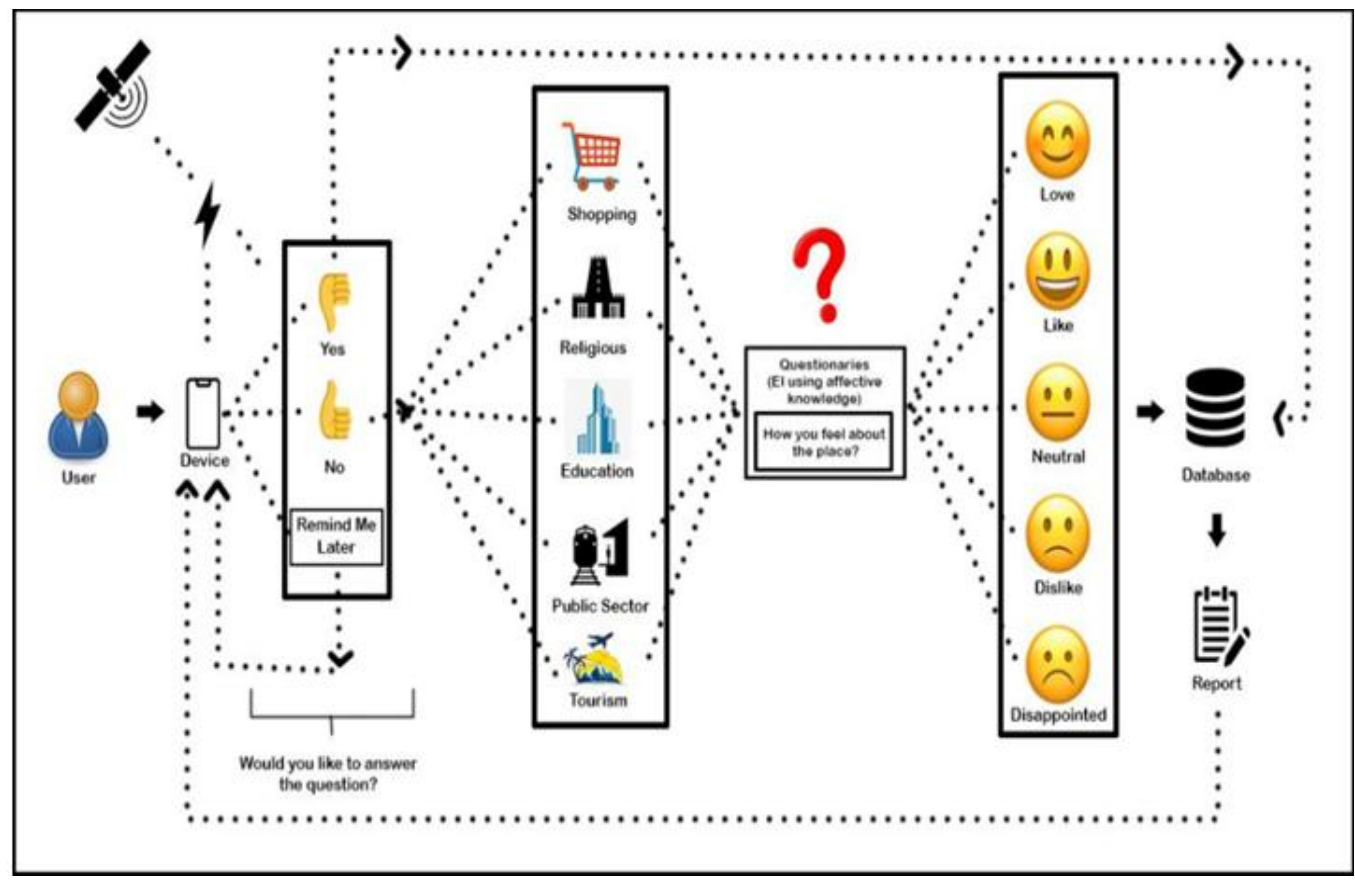

Fig. 1 Architecture of Proposed Work

Initially the user would have to install the application. Then the existing users would be traced to know of their interest for sharing their opinion about a particular location. Based on multinomial distribution just a single question would be asked from any one among the varying categories such as business, shopping, religious, education, public sector etc. An existing user can provide review by clicking on an apt emoticon. These details will be stored in the database. These databases will be optimized based on Emotional Intelligence using affective knowledge and factual knowledge. Thus rankings would be estimated based on the feedback given by the existing user. On viewing the auto generated report, if the new user let out their wish to know more about a particular service that would also be provided capturing up the suggestions given by the existing user. If any existing user were found not interested in answering or if they select remind me later option that information would be stored in the database to be manipulated in the report.

\subsection{Functionalities of this research}

A sequential flow describing the functionalities of this simple yet innovative research is shown in figure 2 .
Location using GPS

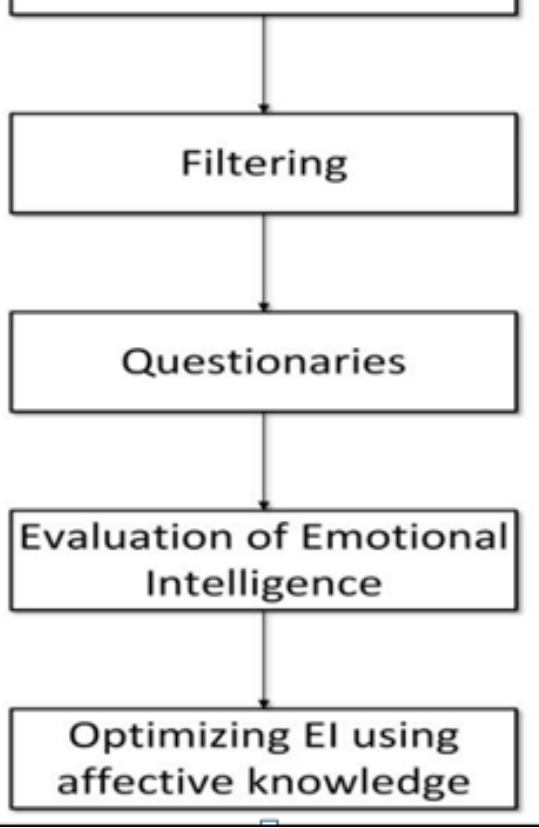

Fig. 2 Functionalities of Proposed work

\subsubsection{Importance of GPS:}

First and foremost is the function of GPS that simplifies the process of identifying a location as per the need of the user, as it tracks and monitors the devices based on the particular location in the real world. GPS is mainly used to track only the mobile devices in which the application is installed and then other geological locations as per the user's choice. 


\subsubsection{Filtering:}

On detecting the location of the mobile device, bounded by the principle of multinomial distribution, a single random question in connection to any one among the various categories such as business, education, religion or public places would be displayed.

\subsubsection{Questionnaire:}

Questionnaires are classified into two categories, initially, first question would be to determine existing user's interest to answer the questionnaire and the second questionnaire would determine the emotional intelligence of that user.

\subsubsection{Evaluation of Emotional intelligence:}

The evaluation of emotional intelligence can be done by analyzing the feedback given by the existing user, based on the multinomial distribution using trait anxiety and emotional intelligence with affective, factual knowledge.

\subsubsection{Optimizing Emotional Intelligence using affective} knowledge:

The feedback provided by the existing users, are manipulated based on the traits of the existing user and this going hand in hand with emotional intelligence using affective knowledge leads the process very much forward to provide the necessary services to the new user.

\subsection{Implementation of proposed Algorithm}

Algorithm for affective knowledge using multinomial distribution. A generalized algorithm of binomial distribution using categorical distribution. Let $\boldsymbol{l}_{\boldsymbol{l}}, \boldsymbol{l}_{2}$ be the latitude and longitude of any geo location of the user.

Let be the global position of the user using GPS. Assume that can be categorized as

\section{Let}

Where possible outcome which is mutual exclusive, with the probabilities $\boldsymbol{\&}$ independent trial. Where the value of smiley selected is based on the category to predict affective knowledge of particular location.

\section{RESULTS AND DISCUSSION}

\subsection{Emotional Intelligence with Affective Knowledge}

Let us implement above proposed algorithm with the sample data to know about St. Peters University, Avadi, Chennai, and Tamil Nadu. When the user enter the location of St. Perters University, the current latitude and longitude of the GPS location are monitored and tracked and provide sample questionnaires based on St. Peters Location. The user responds to the questionnaires via smiles and not as text or documents. This response will be taken into an account and stored in the cloud with hybrid security. The sample data are shown in the below figure 3.

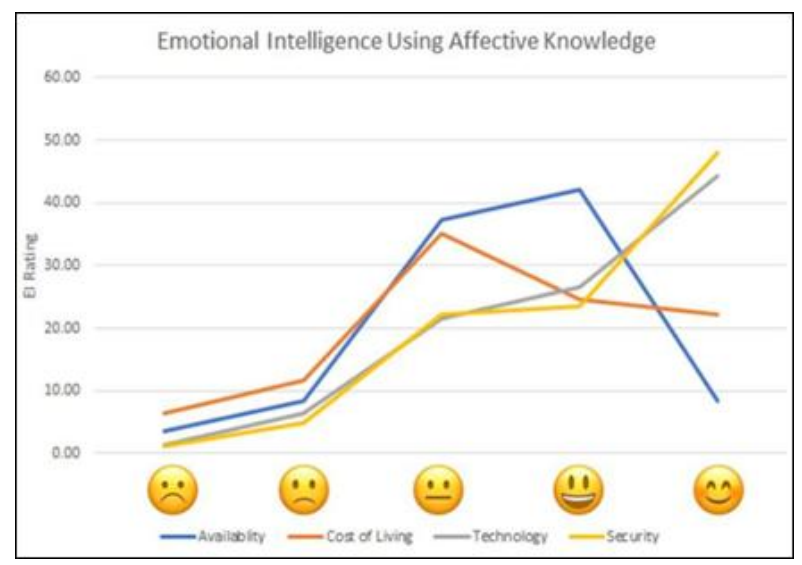

\section{Fig. 3 Emotional Intelligence using Affective} Knowledge

In this proposed work categories are classified into five such as availability, cost of living, education, technology and security. More than five similes to be implemented in this proposed work. Since data are stored in terms of simile's via categories as mention above based on multinomial distribution algorithm. Option for single questions may have more than two options since its leads to multinomial distribution algorithms. Number of user answering the question must also be calculated which reflects in terms of percentage of each simile's.

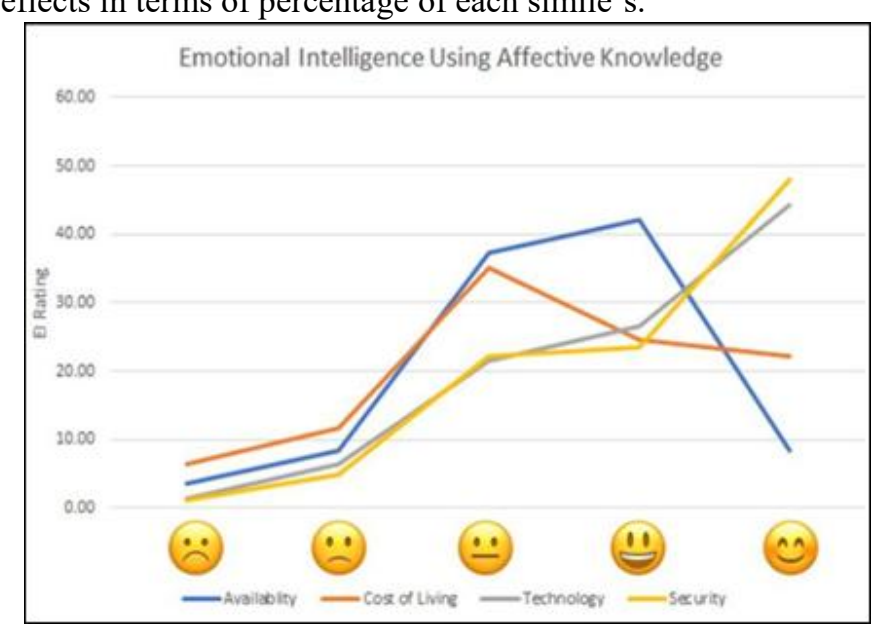

Fig. 4 Feedback Report of Particular Locations

Consider the above graph figure 4 reflect that categories are reflects in $\mathrm{x}$-axis and rating in terms of opinion ranking by the number customer who had answered the questions. The person who skips the questionnaire is not accountable. So based on the sample data of the proposed work are reflects in figure 4. From the Graph generated by the proposed work which provides the information about the particular location based on answer provide by the customer. Customer can take decision based on the probability information provided by the existing customer.

\subsection{Emotional Intelligence using Trait anxiety}

Initially existing user would respond to the questionnaire based on their emotions. A sample data are collected and determine the manipulated data based on the existing user response are as follows figure 5. 


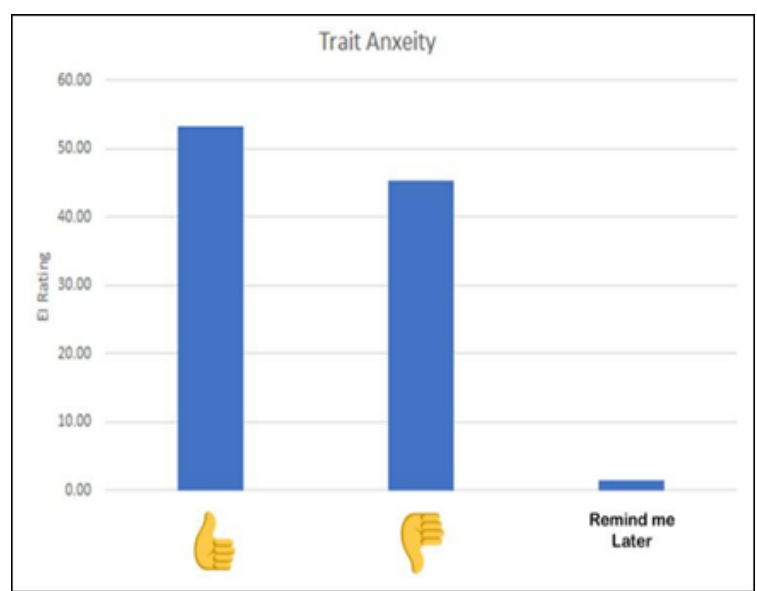

Fig. 5 Trait Anxiety

\subsection{Comparative Study}

a) Entropy based ranking system which provides a several approaches based on Entropy $(\mathrm{H})$, Decision Trees (DT) and National Language Process (NLP) to evaluating the helpfulness of a review, rank product based on relevance from the reviews, enable prediction on the products.

b) Emotional Intelligence, where the user can provide the necessary feedback based on the emoji, which reflects necessary information based on multinomial distribution.

\section{Comparison:}

- $\quad$ More process such as entropy, decision tree and NLP had to be calculated for individual process where as in Emotional Intelligence are flexible.

Implementation of Big data is tedious process in entropy ranking system where as its effective and efficient in Emotional Intelligence.

$\bullet$

Memory utilization will be more compare to emotional intelligence.

- $\quad$ Entropy ranking system mainly focused on text with reflects a hurt the appropriate user where as it's not reflect in emotional intelligence.

- Time take will be short in EI rather than entropy ranking system.

\section{CONCLUSIONS}

Thus framed was the above explained algorithm to be implemented by developing, a smart application. To start with its usage, those who are interested should install it in their device and register giving the essential details. At any moment of need to use it, GPS captures up with the new user's device location and their necessity to get hints about that location. Sifting the stored data the existing users with an experience about that spot are sent a question for understanding their interest to give a feedback on the needed location and if that's positive, they would be requested to reply by clicking on the apt smiley faces for one random question under any of the varying categories in connection to the tracked location. Thus the needed information about a location is received, analyzed and derived from feedbacks, devoid of text and by just clicking on appropriate smiley faces using the principle behind emotional intelligence and multinomial distribution.
These results will be displayed appropriately in the device to help the new or the needed customer to provide the information about the specified location. This would be of a great use mainly to understand a place or an organization or human resources. This work of endeavor with acumen is expected to be a field of attraction for upcoming scholars with perspicacity to enhance this artificial intelligence using neural network and GPS with higher accuracy using private cloud security by bringing in automatic voice recording system without questionnaires. Future study would even upgrade the technology by receiving the answer for the questionnaires from the artificially emotional intelligent devices (like Alexa) personally linked to appropriate mobile devices by the users.

\section{REFERENCE}

1. Adrian Emiell U. Berbano, Hanz Niccole V. Pengson, Cedric Gerard V. Razon and Kristel Chloe G. Tungcul, "Classification of stress into emotional, mental, physical and no stress using electroencephalogram signal analysis", Journal of Signal and Image Processing Applications (ICSIPA), IEEE International Conference, December 2018.

2. Albert Weichselbraun, Stefan Gindl, Fabian Fischer and Svitlana Vakulenko, "Aspect-Based Extraction and Analysis of Affective knowledge from Social Media Streams", Journal of IEEE Computer Socity, Vol. 17, No.1541-1672/17, PP.80-88, June 2017.

3. Arianna Mencattini, Eugenio Martinelli, Fabien Ringeval, Bjorn Schuller, and Corrado di Natale, "Continuous Estimation of Emotions in Speech by Dynamic Coooperative Speaker Models", Journal of IEEE Transaction on Affective computing, Vol. 8, No.03, PP.314-323, September 2017.

4. Boon Giin Lee, Teak Wei Ching, Boon Leng Lee, Hee Joon Park and Yoon Nyun Kim, "Wearable MobileBased Emotional Response -Monitoring System for Drivers", Journal of IEEE Transaction on human machine systems, Vol. 47, No.5, PP.636-641, October 2017.

5. Krishnaveni.K.S, Rohit R Pai, and Vignesh Iyer, "Faculty rating system based on student feedbacks using sentimental analysis", published in: Advances in Computing, Communications and Informatics (ICACCI), Vol. 10, September 2017.

6. Mahdi Hashemi, "Reusability of the output of MapMatching Algorithm Across Space and Time Through Machine Learning", Journal of IEEE Transactions on Intelligent Transportation Systems, Vol. 18, No. 11, pp.3017-3026, March 2017.

7. Marta Arguedas, Luis Casillas and Fatos Xhafa, "A Fuzzy-Based Approach for Classifying Students' Emotional States in Online Collaborative Work Sign In or Purchase", Published in: (CISIS), Vol. 10, No.2, December 2016.

8. Shoiab Ahmed and Ajit Danti, "A novel approach for Sentimental Analysis and Opinion Mining based on SentiWordNet using web data", Journal of Trends in Automation, Communications and Computing Technology (I-TACT), June 2016.

9. Yichao Ren and Jiayin Tian, "Sentiment analysis of Internet performance data", Published in: Information Technology and Mechatronics Engineering Conference (ITOEC), Vol. 05, December 2017. 
10. Yosuke Sakamoto and Yasufumi Takama, "Proposal of sentiment-based tourist spot recommendation system using RDF database", Published in: Computational Intelligence and Applications (IWCIA), Vol. 10, December 2017.

11. Yue-Jiao Gong, En Chen, Xinglin Zhang, Lionel M. Ni and Jun Zhang, "AntMapper: An Ant Colony-Based Map Matching Approach for Trajectory-Based Applications", Journal of IEEE Transactions on Intelligent Transportation Systems, Vol. 19, No. 2, February 2018. 